\title{
Disponibilidade de nitrogênio pela oxidação do carbono lábil com permanganato de potássio ${ }^{1}$
}

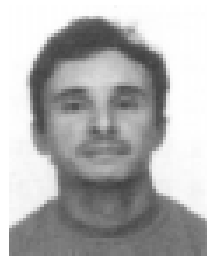

Celsemy E. Maia² \& Reinaldo B. Cantarutti ${ }^{3}$

1 Parte da Dissertação de Mestrado do primeiro autor, apresentada a UFV
2 Doutorando UFCG. CEP 58109-970, Campina Grande, PB. E-mail: celsemymaia@bol.com.br (Foto)
${ }^{3}$ DPS/UFV. CEP 36571-000, Viçosa, MG. Fone: (31) 3899-1052

Protocolo $64-7 / 5 / 2002$ - Aprovado em 12/9/2003

\begin{abstract}
Resumo: O presente trabalho teve como objetivo avaliar a liberação do nitrogênio associado ao carbono lábil oxidado com $\mathrm{KMnO}_{4} 333 \mathrm{mmol} \mathrm{L}^{-1}$ e verificar a capacidade supridora de nitrogênio pela sua correlação com o nitrogênio absorvido pelas plantas. $O$ solo utilizado foi proveniente de um experimento com milho em um Argissolo Vermelho-Amarelo, cultivado com milho desde 1984, cuja produtividade vem sendo avaliada em função das doses de 0 e $40 \mathrm{~m}^{3} \mathrm{ha}^{-1}$ ano ${ }^{-1}$ de composto orgânico (palha de soja e feijão com esterco bovino) combinadas com 0, 250 e 500 $\mathrm{kg} \mathrm{ha}^{-1} \mathrm{ano}^{-1}$ da fórmula 4-14-8 aplicados no plantio, e 0, 100 e $200 \mathrm{~kg} \mathrm{ha}^{-1} \mathrm{ano}^{-1}$ de sulfato de amônio em cobertura. Os resultados mostraram que o uso da matéria orgânica contribuiu para uma quantidade maior de nitrogênio solúvel em $\mathrm{KMnO}_{4} 333 \mathrm{mmol} \mathrm{\textrm {L } ^ { - 1 }}$; o teor de nitrogênio solúvel em $\mathrm{KMnO}_{4} 333 \mathrm{mmol} \mathrm{L}^{-1}$ foi superior ao obtido pelo método anaeróbico e se correlacionou significativamente com o carbono total, carbono lábil, nitrogênio total, teor de amônio e nitrato e com o nitrogênio biodisponível.
\end{abstract}

Palavras-chave: carbono orgânico, oxidação do carbono, experimento de longa duração, adubação contínua

\section{Availability of nitrogen by the oxidation of the labile carbon with potassium permanganate}

\begin{abstract}
The present work had the objective of evaluating the liberation of the nitrogen associated to the labile carbon oxidized with $\mathrm{KMnO}_{4}\left(333 \mathrm{mmol} \mathrm{L}^{-1}\right)$ and to verify the supply capacity of nitrogen by its correlation with the nitrogen absorbed by the plants. The soil used belongs to a Cambic Yellow Red Podzol, cultivated with corn since 1984, and its productivity is being evaluated as a function of the doses of 0 and $40 \mathrm{~m}^{3}$ ha $^{-1}$ year ${ }^{-1}$ of organic compost (beans and soybean straw with manure), combined with 0,250 and $500 \mathrm{~kg} \mathrm{ha}^{-1}$ year $^{-1}$ of the formula 4-14-8 applied at planting and 0,100 and $200 \mathrm{~kg} \mathrm{ha}^{-1}$ year $^{-1}$ of ammonium sulphate as top dressing. The results showed that the use of the organic material contributed to a higher amount of nitrogen soluble in $\mathrm{KMnO}_{4}\left(333 \mathrm{mmol} \mathrm{L}^{-1}\right)$. The content of soluble nitrogen in $\mathrm{KMnO}_{4}$ (333 $\mathrm{mmol} \mathrm{L}^{-1}$ ) was found to be superior than that obtained by the anaerobic method and correlated significantly with the total carbon, labile carbon, total nitrogen, ammonium and nitrate content and with the nitrogen bioavailability.
\end{abstract}

Key words: organic carbon, oxidation of the carbon, long-term experiment, continuous fertilization

\section{INTRODUÇÃO}

A aplicação de doses adequadas de $\mathrm{N}$ é um dos fatores que determinam a eficiência da adubação nitrogenada. Para isso, a capacidade natural do solo em suprir este nutriente precisa ser devidamente considerada. A disponibilidade de $\mathrm{N}$ para as plantas depende tanto do teor desse nutriente imediatamente disponível, representada pelas formas inorgânicas, quanto do $\mathrm{N}$ das formas orgânicas, mineralizáveis durante o seu ciclo de vida (Brandão, 1990).

Segundo Vidor et al. (1995) a maioria dos estudos envolvendo a mineralização do $\mathrm{N}$ do solo caracteriza-se por experimentos de curta duração, justificados pela necessidade de métodos rápidos e adequados para a avaliação da disponibilidade de $\mathrm{N}$. Nessas condições, somente uma parte do N potencialmente mineralizável é liberada, resultando em provável subestimação do $\mathrm{N}$ potencialmente mineralizável.

O N potencialmente mineralizável do solo é uma medida da quantidade de $\mathrm{N}$ mineralizado sob condições ótimas de umidade e temperatura; assim, pode ser empregado para estimar o $\mathrm{N}$ que será disponibilizado durante o ciclo de uma cultura. No período de crescimento das plantas, existe variação nas quantidades de $\mathrm{N}$ inorgânico (principalmente $\mathrm{NO}_{3}^{-}$) e também na capacidade que o solo tem de fornecer $\mathrm{N}$ às plantas por 
meio da mineralização. A contribuição relativa das formas orgânicas e minerais varia de acordo com o tipo de solo e o sistema de manejo (Stanford \& Smith, 1976).

Inúmeros são os esforços em prol do desenvolvimento de modelos para simular a dinâmica da matéria orgânica do solo. Geralmente, esses modelos se aproximam das variações que ocorrem no solo, quando várias frações da matéria orgânica, que diferem pela qualidade e, conseqüentemente, pela sua taxa de decomposição, são incorporadas aos modelos.

Swift et al. (1991) citados por Blair et al. (1995) argumentam que o manejo sustentável da matéria orgânica no solo está baseado em duas suposições, em que a primeira, de que a matéria orgânica é separada em frações, em que cada uma responde de forma diferente ao manejo e às práticas do uso do solo e a segunda é a de que a decomposição e a síntese de cada uma das frações são reguladas por processos físicoquímicos e biológicos que podem ser alterados pelo manejo. Um método comumente usado na caracterização da matéria orgânica é o fracionamento químico, obtendo-se as frações ácido fúlvico, ácido húmico e humina, com base na solubilidade dos compostos orgânicos em meio ácido e em meio alcalino; entretanto, essas frações nem sempre se correlacionam com as características da matéria orgânica do solo. Outro tipo de fracionamento da matéria orgânica combina princípios físicos e químicos. Para este fim, é feita a separação física das frações da matéria orgânica por meio do peneiramento e pela diferença de densidade através da flotação em líquidos densos, seguida de análise química de cada fração.

De acordo com Loginow et al. (1987) o processo de separação de frações orgânicas do solo com base na solubilidade em ácido ou base, parece não ser adequado do ponto de vista da avaliação das transformações da matéria orgânica nos solos. A utilização da biomassa microbiana para avaliar a suscetibilidade da matéria orgânica à decomposição, é seguramente o método mais apropriado, mas requer procedimentos laboriosos. Esta situação induziu os pesquisadores à busca de uma metodologia simples, que se correlacionasse com a decomposição microbiana.

Conceitualmente, carbono lábil é aquele carbono constituinte de compostos orgânicos mais facilmente mineralizados pelos microrganismos do solo. Operacionalmente, pode ser considerado o carbono oxidável com uma solução de $\mathrm{KMnO}_{4}$ $333 \mathrm{mmol} \mathrm{L}^{-1}$, visto que este se correlaciona com o carbono oxidado pelos microrganismos do solo, medido pela evolução do $\mathrm{CO}_{2}$ (Loginow et al., 1987). Esta correlação se justifica pelo fato da decomposição microbiana da matéria orgânica no solo se processar por oxidação de caráter enzimático. Este processo foi inicialmente desenvolvido por Loginow et al. (1987) e modificado posteriormente por Blair et al. (1995) e Shang \& Tiessen (1997).

O objetivo deste trabalho foi avaliar a liberação do nitrogênio associado ao carbono lábil oxidado com $\mathrm{KMnO}_{4}$ $333 \mathrm{mmol} \mathrm{L}^{-1}$ e verificar a capacidade supridora de nitrogênio pela sua correlação com o nitrogênio absorvido pelas plantas.

\section{MATERIAL E MÉTODOS}

\section{Caracterização do solo}

Utilizaram-se, para o estudo, amostras de solo coletadas em uma área na Estação Experimental de Coimbra, pertencente à Universidade Federal de Viçosa, localizada no município de Coimbra ( $\left.20^{\circ} 45^{\prime} \mathrm{S} ; 42^{\circ} 51^{\prime} \mathrm{O}\right)$ na Zona da Mata mineira. O solo da área experimental é um Argissolo Vermelho-Amarelo fase terraço, com $80 \mathrm{~g} \mathrm{~kg}^{-1}$ de areia grossa, $50 \mathrm{~g} \mathrm{~kg}^{-1}$ de areia fina, $170 \mathrm{~g} \mathrm{~kg}^{-1}$ de silte e $700 \mathrm{~g} \mathrm{~kg}^{-1}$ de argila, sendo classificado como muito argiloso. A área já tinha sido cultivada durante 13 anos com a cultura do milho em um experimento em fatorial e a produção tem sido avaliada sob as doses de 0 e $40 \mathrm{~m}^{3} \mathrm{ha}^{-1}$ ano $^{-1}$ de composto orgânico, combinadas com três doses de adubo químico, ao longo de 13 cultivos. As adubações químicas correspondem a 0,250 e $500 \mathrm{~kg} \mathrm{ha}^{-1}$ da fórmula 4-14-8 aplicados no plantio. Em cobertura aplicaram-se, em cada cultivo, 0, $20 \mathrm{e}$ $40 \mathrm{~kg} \mathrm{ha}^{-1}$ de $\mathrm{N}$ na forma de sulfato de amônio.

\section{Ensaio biológico em casa de vegetação}

As unidades experimentais foram constituídas por vasos contendo $2,5 \mathrm{dm}^{3}$ de solo, provenientes de cada parcela do experimento de campo. As unidades experimentais foram distribuídas em blocos casualizados, mantendo-se as quatro repetições de campo.

O solo de cada unidade recebeu uma adubação complemen$\operatorname{tar} \operatorname{com} 300,150$ e $40 \mathrm{mg} \mathrm{dm}^{-3} \mathrm{de} \mathrm{P}\left(\mathrm{CaHPO}_{4}\right), \mathrm{K}\left(\mathrm{KCle} \mathrm{K}_{2} \mathrm{SO}_{4}\right) \mathrm{e}$ $\mathrm{S}\left(\mathrm{K}_{2} \mathrm{SO}_{4}\right)$ respectivamente, assim como 4,0, 1,55, 3,66, 1,33 e $0,81 \mathrm{mg} \mathrm{dm}^{-3} \mathrm{de} \mathrm{Zn}\left(\mathrm{ZnCl}_{2}\right), \mathrm{Fe}\left(\mathrm{FeCl}_{3}\right), \mathrm{Mn}\left(\mathrm{MnCl}_{2}\right), \mathrm{Cu}\left(\mathrm{CuCl}_{2}\right)$ e B $\left(\mathrm{H}_{3} \mathrm{BO}_{3}\right)$, respectivamente (Novais et al., 1991). Para isto, utilizou-se uma solução nutritiva aplicada e homogeneizada com o volume de solo de cada vaso, antes do plantio. Aos 15 dias após o plantio, foram aplicados mais $50 \mathrm{mg} \mathrm{dm}^{-3} \mathrm{de} \mathrm{P}$ na forma de $\mathrm{NaH}_{2} \mathrm{PO}_{4}$, devido ao surgimento da deficiência de fósforo nas plantas de milho.

Foram plantadas 10 sementes de milho híbrido C-435, deixando-se cinco plantas por vaso após o desbaste, que foi realizado seis dias após o plantio. A duração do experi-mento foi de 40 dias, contando desde o plantio das sementes. Durante esse período, a umidade do solo foi mantida em torno de $80 \%$ da capacidade de campo, com irrigações periódicas com água destilada.

Ao final desse período, as plantas foram cortadas rente ao solo, sendo o material acondicionado em saco de papel e submetido a secagem, em estufa de circulação forçada a $65^{\circ} \mathrm{C}$, até peso constante. A matéria seca do tecido vegetal foi pesada e, depois, moída para ser analisada. Após sofrer a digestão sulfúrica, o $\mathrm{N}$ total na matéria seca foi determinado pelo método da destilação Kjeldahl, descrito por Tedesco et al. (1985).

\section{Análises químicas}

Nas amostras mantidas com umidade natural, as formas inorgânicas de nitrogênio $\left(\mathrm{NH}_{4}^{+} \mathrm{e} \mathrm{NO}_{3}^{-}\right)$foram extraídas com $\mathrm{KCl}$ 1,0 mol L-1 utilizando-se relação solo:solução de 1:10 e agitação por uma hora. No filtrado, foram determinados o $\mathrm{NH}_{4}^{+}$ e o $\mathrm{NO}_{3}^{-}$sendo o amônio pela destilação Kjeldhal (Keeney \& Nelson, 1982) . $\mathrm{O} \mathrm{NO}_{3}$ - foi determinado indiretamente após sua redução a $\mathrm{NO}_{2}^{-}$, empregando-se sulfato de hidrazina, sendo este dosado colorimetricamente, com base na reação GriessIlosvay, conforme descrito por Keeney \& Nelson (1982). Os resultados foram corrigidos para peso de solo seco a ar.

Nas subamostras foram determinados o nitrogênio total, o nitrogênio mineralizável anaerobicamente (NMA), o carbono 
total, o carbono oxidável em $\mathrm{KMnO}_{4} 333 \mathrm{mmol} \mathrm{L}^{-1}$ (C lábil) e o nitrogênio solúvel em $\mathrm{KMnO}_{4} 333 \mathrm{mmol} \mathrm{L}^{-1}$.

O nitrogênio total foi determinado em $0,5 \mathrm{~g}$ de solo passada em peneira de $0,15 \mathrm{~mm}$ (100 mesh) após ter sido triturada em almofariz. O solo foi submetido a digestão sulfúrica e o nitrogênio dosado por destilação Kjeldahl (Bremner \& Mulvaney, 1982).

O nitrogênio mineralizável anaerobicamente (NMA) foi determinado pelo método da incubação anaeróbica desenvolvido por Waring \& Bremner (1964) adotando-se o procedimento descrito por Keeney (1982). Para tanto, $5 \mathrm{~g}$ de solo foi colocado em tubo de vidro $(16 \times 150 \mathrm{~mm})$ adicionandose $12 \mathrm{~mL}$ de água. Os tubos foram tampados com rolha de borracha, para evitar a entrada de ar, e incubados a $35^{\circ} \mathrm{C}$ pelo período de sete dias. Após a incubação, os tubos foram agitados e a mistura solo-água transferida para tubos de destilação, juntamente com $15 \mathrm{~mL}$ de $\mathrm{KCl} 1,0 \mathrm{~mol} \mathrm{~L}^{-1}$ e aproximadamente $0,3 \mathrm{~g}$ de $\mathrm{MgO} . \mathrm{O} \mathrm{NH}_{3}$ liberado foi imediatamente destilado e o $\mathrm{NH}_{4}{ }^{+}$dosado conforme metodologia descrita por Keeney \& Nelson (1982). Determinou-se o $\mathrm{NH}_{4}^{+}$inicialmente presente no solo e, por diferença, foi calculada a quantidade de $\mathrm{NH}_{4}^{+}$ produzida durante a incubação.

O carbono total foi determinado em amostras de solo trituradas em almofariz e passada em peneira de $0,5 \mathrm{~mm}$, pelo método de Walkley-Black, citado por Defelipo \& Ribeiro (1981). Por sua vez, o carbono facilmente oxidado, caracterizado como carbono lábil, foi determinado conforme procedimento descrito por Blair et al. (1995). Uma amostra de solo passada em peneira de $0,5 \mathrm{~mm}$, contendo aproximadamente $25 \mathrm{mg}$ de $\mathrm{C}$ orgânico, foi colocada em tubos de centrífuga de $30 \mathrm{~mL}$, adicionando-se $25 \mathrm{~mL}$ de solução de $\mathrm{KMnO}_{4} 333 \mathrm{mmol} \mathrm{L}^{-1}$. Os tubos foram tapados e agitados por uma hora, em agitador vertical a $12 \mathrm{rpm}$; em seguida, eles foram centrifugados a $2.000 \mathrm{rpm}$ por cinco min, e 1,0 mL do sobrenadante foi transferido para um balão volumétrico de $250 \mathrm{~mL}$, completando-se o volume com água destilada. Alíquotas de 1,0 mL de seis soluções-padrões de $\mathrm{KMnO}_{4}$, com concentrações variando de 280 a $333 \mathrm{mmol} \mathrm{L}^{-1}$, sofreram a mesma diluição. Determinou-se a absorvância das soluções diluídas em espectrofotômetro ajustado para o comprimento de onda de $565 \mathrm{~nm}$. A variação na concentração do $\mathrm{KMnO}_{4}$, estimada a partir da curva-padrão, foi usada para se estimar a quantidade de $\mathrm{C}$ oxidado (C lábil) considerando-se que 1,0 mol de $\mathrm{MnO}_{4}$ - é consumido na oxidação de 0,75 mol (9 g) de carbono.

Para determinação do nitrogênio solúvel em $\mathrm{KMnO}_{4} 333$ mmol L-1, $20 \mathrm{~mL}$ do sobrenadante centrifugado foram submetidos a digestão sulfúrica, e o nitrogênio foi dosado por destilação Kjeldahl (Bremner \& Mulvaney, 1982).

\section{Análises estatísticas}

Os dados foram submetidos a análise de variância e análise de correlação (Pearson) utilizando-se o sistema computacional SAEG (Sistema de Análise Estatística e Genética) desenvolvido pela Universidade Federal de Viçosa. Para a análise de variância, as duas profundidades de amostragem foram consideradas isoladamente e as correlações foram realizadas com os valores médios das duas profundidades.

\section{RESULTADOS E DISCUSSÃO}

A solução diluída de $\mathrm{KMnO}_{4}$ tem sido empregada para se estimar a fração mais facilmente oxidável de C, sendo denominada C lábil (Blair et al., 1995; Shang \& Tiessen, 1997). Dentro desse princípio, o $\mathrm{N}$ liberado durante a oxidação do $\mathrm{C}$ pode constituir-se em um estimador do $\mathrm{N}$ potencialmente mineralizável ou, em outras palavras, da capacidade supridora de $\mathrm{N}$ no solo.

O N solúvel em $\mathrm{KMnO}_{4} 333 \mathrm{mmol} \mathrm{L}^{-1}$ foi, em média, maior nos tratamentos com adubação orgânica nas camadas de 0 a 10 e 10 a $20 \mathrm{~cm}$, com os maiores teores verificados na camada superficial, Tabela 1. Nos tratamentos que receberam adubação orgânica, esses teores foram, em média, 41 e $32 \%$ superiores nas profundidades de 0 a 10 e 10 a $20 \mathrm{~cm}$, respectivamente. $\mathrm{O}$ maior teor de $\mathrm{N}$ solúvel em $\mathrm{KMnO}_{4} 333 \mathrm{mmol} \mathrm{L}^{-1}$ nos tratamentos que receberam adubação orgânica é explicado pelos maiores teores de matéria orgânica e por sua labilidade, Tabela 1.

Na camada de 0 a $10 \mathrm{~cm}$, o N solúvel em $\mathrm{KMnO}_{4} 333 \mathrm{mmol} \mathrm{L}^{-1}$ correspondeu, em média, a 13,5 e 13,7\% do $\mathrm{N}$ total para os tratamentos sem e com adubação orgânica, respectivamente; já na profundidade de 10 a $20 \mathrm{~cm}$, esses valores foram

Tabela 1. Teor de $\mathrm{N}$ solúvel em $\mathrm{KMnO}_{4} 333 \mathrm{mmol} \mathrm{L}^{-1}\left(\mathrm{~N}-\mathrm{KMnO}_{4}\right)$, carbono total $(\mathrm{C}$ Total), carbono lábil (C Lábil) e nitrogênio total (N Total) em amostras de 0 a 10 e 10 a 20 cm, de um Argissolo Vermelho-Amarelo, em função das adubações orgânica e mineral, em 13 cultivos sucessivos de milho

\begin{tabular}{|c|c|c|c|c|c|c|c|c|c|c|c|}
\hline \multicolumn{2}{|c|}{ Adubação } & \multicolumn{4}{|c|}{$\mathrm{N}-\mathrm{KMnO}_{4}$} & \multicolumn{2}{|c|}{ C Total } & \multicolumn{2}{|c|}{ C Lábil ${ }^{2}$} & \multicolumn{2}{|c|}{ N Total } \\
\hline \multirow{2}{*}{$\begin{array}{c}\text { Orgânica } \\
\mathrm{m}^{3} \mathrm{ha}^{-1} \text { ano }^{-1}\end{array}$} & \multirow{2}{*}{$\begin{array}{c}\text { Mineral } \\
\mathrm{kg} \mathrm{ha}^{-1} \text { ano }^{-1}\end{array}$} & \multicolumn{2}{|c|}{$0-10$} & \multicolumn{2}{|c|}{$10-20$} & $0-10$ & $10-20$ & $0-10$ & $10-20$ & $0-10$ & $10-20$ \\
\hline & & $\mathrm{mg} \mathrm{kg}^{-1}$ & $\%^{2}$ & $\mathrm{mg} \mathrm{kg}^{-1}$ & $\%^{2}$ & \multicolumn{6}{|c|}{ dag $\mathrm{kg}^{-1}$} \\
\hline \multirow{3}{*}{0} & 0 & 212,76 & 13,30 & 169,31 & 11,29 & 2,61 & 2,30 & 0,72 & 0,83 & 0,16 & 0,15 \\
\hline & $250(20)$ & 227,74 & 13,40 & 186,09 & 11,63 & 2,73 & 2,59 & 0,74 & 0,75 & 0,17 & 0,16 \\
\hline & $500(40)$ & 222,04 & 13,88 & 170,80 & 11,39 & 2,67 & 2,31 & 0,64 & 0,68 & 0,16 & 0,15 \\
\hline Média & & $220,85 B$ & 13,53 & $75,40 \mathrm{~B}$ & 11,44 & $2,67 \mathrm{~B}$ & $2,40 \mathrm{~B}$ & $0,70 \mathrm{~B}$ & $0,75 \mathrm{~B}$ & $0,16 \mathrm{~B}$ & $0,15 \mathrm{~B}$ \\
\hline \multirow{3}{*}{40} & 0 & 306,55 & 13,93 & 233,13 & 12,27 & 3,26 & 2,95 & 0,97 & 0,96 & 0,22 & 0,19 \\
\hline & $250(20)$ & 326,03 & 13,58 & 227,74 & 11,39 & 3,35 & 2,88 & 0,96 & 0,92 & 0,24 & 0,20 \\
\hline & $500(40)$ & 300,56 & 13,66 & 231,64 & 12,19 & 3,26 & 2,81 & 0,91 & 0,99 & 0,22 & 0,19 \\
\hline Média & & $311,05 \mathrm{~A}$ & 13,72 & $230,84 \mathrm{~A}$ & 11,95 & $3,29 \mathrm{~A}$ & $2,88 \mathrm{~A}$ & $0,95 \mathrm{~A}$ & $0,95 \mathrm{~A}$ & $0,23 \mathrm{~A}$ & $0,19 \mathrm{~A}$ \\
\hline CV (\%) & & 10,84 & - & 7,77 & - & 10,65 & 8,85 & 18,02 & 13,34 & 8,81 & 8,04 \\
\hline
\end{tabular}

Valores fora dos parênteses correspondem a $\mathrm{kg} \mathrm{ha}^{-1}$ ano $^{-1}$ da fórmula 4-14-8 aplicada no plantio e, dentro, a $\mathrm{kg} \mathrm{ha}^{-1}$ ano ${ }^{-1}$ de $\mathrm{N}$ na forma de sulfato de amônio aplicado em cobertura

${ }^{2} \mathrm{C}$ oxidado pela solução de $\mathrm{KMnO}_{4} 333 \mathrm{mmol} \mathrm{L}^{-1}$, conforme metodologia descrita por BLAIR et al. (1995)

- Para cada profundidade, as médias com letras diferentes, na mesma coluna, não diferem estatisticamente pelo teste $\mathrm{F}$, a $5 \%$ de probabilidade 
de 11,4 e $11,9 \%$ do $\mathrm{N}$ total. Observa-se que os teores de $\mathrm{N}$ solubilizados em $\mathrm{KMnO}_{4}$, tanto em termos absolutos como relativos ao $\mathrm{N}$ total (Tabela 1), foram maiores do que o $\mathrm{N}$ produzido pela oxidação microbiana sob condições anaeróbicas (Tabela 2), isto é explicado pelo fato da oxidação com o $\mathrm{KMnO}_{4}$ ser mais forte que a oxidação produzida pelos microrganismos anaeróbicos; além disso, ao contrário do verificado com o NMA, uma proporção mais uniforme do $\mathrm{N}$ total foi oxidada pelo $\mathrm{KMnO}_{4}$.

Tabela 2. Teor de $\mathrm{N}$ mineralizado obtido por incubação anaeróbica (NMA) de amostras das camadas de 0 a 10 e 10 a $20 \mathrm{~cm}$, de um Argissolo Vermelho-Amarelo, em função das adubações orgânica e mineral anual, aplicadas em 13 cultivos sucessivos de milho

\begin{tabular}{|c|c|c|c|c|c|}
\hline \multicolumn{2}{|c|}{ Adubação } & \multicolumn{4}{|c|}{ NMA } \\
\hline \multirow{2}{*}{$\begin{array}{l}\text { Orgânica } \\
\mathrm{m}^{3} \mathrm{ha}^{-1} \text { ano }^{-1}\end{array}$} & \multirow{2}{*}{$\begin{array}{c}\text { Mineral }^{1} \\
\mathrm{~kg} \mathrm{ha}^{-1} \mathrm{ano}^{-1}\end{array}$} & \multicolumn{2}{|c|}{$0-10$} & \multicolumn{2}{|c|}{$10-20$} \\
\hline & & $\mathrm{mg} \mathrm{kg}^{-1}$ & $\%^{2}$ & $\mathrm{mg} \mathrm{kg}^{-1}$ & $\%^{2}$ \\
\hline \multirow{3}{*}{0} & 0 & 30,17 & 1,89 & 39,73 & 2,65 \\
\hline & $250(20)$ & 52,74 & 3,10 & 45,35 & 2,83 \\
\hline & $500(40)$ & 62,12 & 3,88 & 48,26 & 3,22 \\
\hline Média & & $48,34 \mathrm{~B}$ & 2,79 & 44,44B & 2,90 \\
\hline \multirow{3}{*}{40} & 0 & 56,72 & 2,58 & 58,88 & 3,10 \\
\hline & $250(20)$ & 77,27 & 3,22 & 56,66 & 2,83 \\
\hline & $500(40)$ & 79,12 & 3,60 & 66,72 & 3,51 \\
\hline Média & & $71,04 \mathrm{~A}$ & 3,13 & $60,75 \mathrm{~A}$ & 3,15 \\
\hline $\mathrm{CV}(\%)$ & & 29,56 & & 32,00 & \\
\hline
\end{tabular}

Valores fora dos parênteses correspondem a $\mathrm{kg} \mathrm{ha}^{-1} \mathrm{ano}^{-1}$ da fórmula 4-14-8 aplicada no plantio e, dentro, a kg ha- ${ }^{-1}$ ano $^{-1}$ de $\mathrm{N}$ aplicado como sulfato de amônio em cobertura

Porcentagem em relação ao $\mathrm{N}$ total

- Médias com letras diferentes, na mesma coluna, diferem estatisticamente pelo teste $\mathrm{F}$, a $5 \%$ probabilidade

Segundo Loginow et al. (1987) existe correlação entre a oxidação química realizada pelo $\mathrm{KMnO}_{4}$ e a oxidação de caráter enzimático responsável pela mineralização da matéria orgânica do solo. Assim, os tratamentos que receberam $40 \mathrm{~m}^{3} \mathrm{ha}^{-1} \mathrm{ano}^{-1}$ de composto orgânico, têm maior potencial para fornecer $\mathrm{N}$ às plantas.

Avaliando a labilidade da matéria orgânica em um Oxisol de clima tropical, Shang \& Tiessen (1997) verificaram que o carbono oxidado pelo $\mathrm{KMnO}_{4} 333 \mathrm{mmol} \mathrm{L}^{-1}$ correspondeu a aproximadamente $50 \%$ do carbono total do solo, tanto em um solo cultivado como em um solo sob vegetação nativa. Esses autores concluíram que esta oxidação não foi sensível à variabilidade da agregação nem à estabilidade da matéria orgânica entre os solos. Usando-se, no entanto, a concentração de $33 \mathrm{mmol} \mathrm{L}^{-1}$, o teor de carbono oxidado foi de aproximadamente $25 \%$ do carbono total; verificou-se, então, que houve melhor diferenciação entre o solo cultivado e o não-cultivado, refletindo as alterações qualitativas na matéria orgânica no solo. Esses autores salientam ainda que os solos de clima tropical apresentam matéria orgânica relativamente mais lábil que os solos de regiões de clima temperado, para os quais o método foi desenvolvido.

Apesar das variações verificadas no teor de $\mathrm{N}$ solúvel em $\mathrm{KMnO}_{4} 333 \mathrm{mmol} \mathrm{L}^{-1}$, a porcentagem do $\mathrm{N}$ total oxidado foi praticamente a mesma, tanto para a profundidade de 0 a 10 como para a de 10 a $20 \mathrm{~cm}$. A exemplo do verificado para o C oxidado, esses resultados sugerem que a utilização de $\mathrm{KMnO}_{4}$ menos concentrado pode aumentar a sensibilidade do método para estimar o $\mathrm{N}$ potencialmente mineralizável.

O N solúvel em $\mathrm{KMnO}_{4}$ também se correlacionou significativamente com $\mathrm{N}$ total e com os indicadores de disponibilidade de $\mathrm{N}\left(\mathrm{NH}_{4}^{+}, \mathrm{NO}_{3}^{-}\right.$e $\mathrm{N}$-biodisponível); no entanto, os coeficientes das correlações foram maiores que os verificados para o NMA (Tabela 3). Considerando-se este fato e, ainda, a correlação significativa entre o NMA e o N-KMnO 4 (Figura 1), sugere-se que o $\mathrm{N}$ solubilizado pelo $\mathrm{KMnO}_{4} 333 \mathrm{mmol} \mathrm{L}^{-1}$ pode constituir-se em um adequado índice de disponibilidade de $\mathrm{N}$.

Tabela 3. Coeficientes de correlação linear simples do $\mathrm{N}$ mineralizado em incubação anaeróbica e do $\mathrm{N}$ solúvel em $\mathrm{KMnO}_{4} 333 \mathrm{mmol} \mathrm{L}^{-1} \mathrm{com}$ as diferentes frações de carbono do solo e os indicadores da reserva e disponibilidade de $\mathrm{N}$ em um Argissolo Vermelho-Amarelo, após 13 cultivos sucessivos de milho com adubações orgânica e mineral

\begin{tabular}{ccc} 
& $\mathrm{NMA}$ & $\mathrm{N}-\mathrm{KMnO}_{4}$ \\
\hline C-total & $0,347^{\mathrm{ns}}$ & $0,924^{* *}$ \\
C-Lábil & $0,307^{\mathrm{ns}}$ & $0,757^{* *}$ \\
$\mathrm{~N}-\mathrm{Total}$ & $0,492^{* *}$ & $0,956^{* *}$ \\
$\mathrm{~N}^{*} \mathrm{NH}_{4}^{+}$ & $0,389^{*}$ & $0,626^{* *}$ \\
$\mathrm{~N}^{-} \mathrm{NO}_{3}$ & $0,373^{*}$ & $0,746^{* *}$ \\
$\mathrm{~N}-$ biodisponível & $0,505^{* *}$ & $0,829^{* *}$ \\
\hline
\end{tabular}

- As correlações foram estabelecidas com os teores médios das duas profundidades , " Significativo a 5\% e $1 \%$ de probabilidade, respectivamente, pelo teste $\mathrm{t}$

${ }^{n s}$ Não-significativo até 5\% de probabilidade

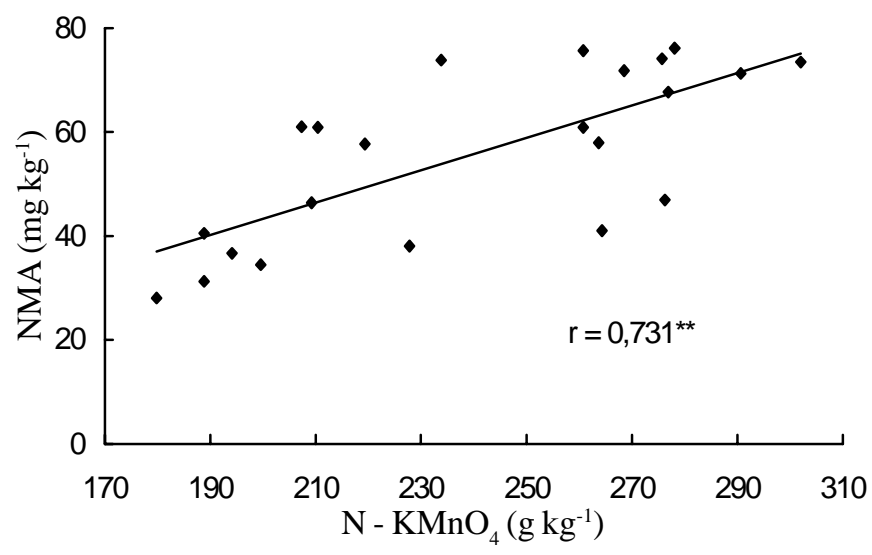

Figura 1. Correlação entre os teores médios de $\mathrm{N}$ solúvel em $\mathrm{KMnO}_{4} 333 \mathrm{mmol} \mathrm{L}^{-1}$ e N mineralizado anaerobicamente (NMA) na camada de 0 a $20 \mathrm{~cm}$ de um Argissolo Vermelho-Amarelo, após 13 cultivos de milho com adubações orgânica e mineral

A maior correlação do $\mathrm{N}-\mathrm{KMnO}_{4}$ com o $\mathrm{C}$ orgânico total que com o $\mathrm{C}$ lábil se deve possivelmente, conforme sugerido por Shang \& Tiessen (1997), ao fato do $\mathrm{KMnO}_{4}$, na concentração empregada, provocar a oxidação de formas menos lábeis de C; assim, um ajuste nessa concentração tornará o método mais adequado para se estimar tanto o $\mathrm{C}$ lábil como a capacidade supridora de $\mathrm{N}$ do solo.

\section{CONCLUSÕES}

1. O uso da matéria orgânica contribuiu para uma quantidade maior de nitrogênio solúvel em $\mathrm{KMnO}_{4} 333 \mathrm{mmol} \mathrm{L}^{-1}$.

2. O teor de nitrogênio solúvel em $\mathrm{KMnO}_{4} 333 \mathrm{mmol} \mathrm{L}^{-1}$ foi superior ao obtido pelo método anaeróbico. 
3. O nitrogênio solúvel em $\mathrm{KMnO}_{4} 333 \mathrm{mmol} \mathrm{L} \mathrm{L}^{-1}$ correlacionou-se significativamente com o carbono total, carbono lábil, nitrogênio total, teor de amônio e nitrato e com o nitrogênio biodisponível.

\section{LITERATURA CITADA}

Blair, G.J.; Lefroy, R.D.B.; Lisle, L. Soil carbon fractions based on their degree of oxidation, and the development of a carbon management index for agricultural system. Australian Journal of Agriculture Research, v.46, p.1459-1466, 1995.

Brandão, R.P. Relação do nitrogênio disponível no solo com o crescimento e absorção do N pelo algodoeiro (Gossypium hirsutum L.). Lavras: UFLA, 1990. 89p. Dissertação Mestrado

Bremner, J.M.; Mulvaney, C.S. Nitrogen total. In: Page, A.L. (ed.) Methods of soil analysis. Part 2. Madison: American Society of Agronomy, 1982. p.595-624.

Defelipo, B.V.; Ribeiro, A.C. Análise química do solo. Viçosa: UFV, 1981. 17p. Boletim de Extensão, 29

Keeney, D.R. Nitrogen - availability indices. In: Page, A.L. Methods of soil analysis. Part 2. Madison: American Society of Agronomy, 1982. p.711-733.

Keeney, D.R.; Nelson, D.W. Nitrogen inorganic forms. In: Page, A.L. (ed.) Methods of soil analysis. Part 2. Madison: American Society of Agronomy, 1982. p.643-698.
Loginow, W.; Wisniewski, W.; Gonet, S.S.; Ciescinska, B. Fractionation of organic carbon based on susceptibility to oxidation. Polish Journal Soil Science, v.20, p.47-52, 1987.

Novais, R.F.; Neves, J.C.L.; Barros, N.F. de. Ensaio em ambiente controlado. In: EMBRAPA. Métodos de pesquisa em fertilidade do solo. Brasília: EMBRAPA, 1991. p.189-251.

Shang, C.; Tiessen, H. Organic matter lability in a tropical oxisol: evidence from shifting cultivation, chemical oxidation, particle size, density, and magnetic fractionations. Soil Science, Baltimore, v.162, p.795-807, 1997.

Stanford, G.; Smith, S.J. Estimating potentially mineralizable soil nitrogen from a chemical index of soil nitrogen availability. Soil Science, Baltimore, v.122, p.71-76, 1976.

Tedesco, J.M.; Wolkweiss, S.J.; Bohnen, H. Análise de solo, planta e outros materiais. Porto Alegre: Universidade Federal do Rio Grande do Sul, 1985. 156p. Boletim Técnico, 5

Vidor, C.; Camargo, F.A.O.; Gianello, C. Variação temporal e espacial em experimentos de incubação para determinação do $\mathrm{N}$ mineralizado. In: Congresso Brasileiro de Ciência do Solo, 25, Viçosa, 1995. Resumo... Viçosa: Sociedade Brasileira de Ciência do Solo, 1995. p.695-696.

Waring, S.A.; Bremner, J.M. Ammonium production in soil under waterlogged conditions as an index of nitrogen availability. Nature, London, v.201, p.951-952, 1964. 